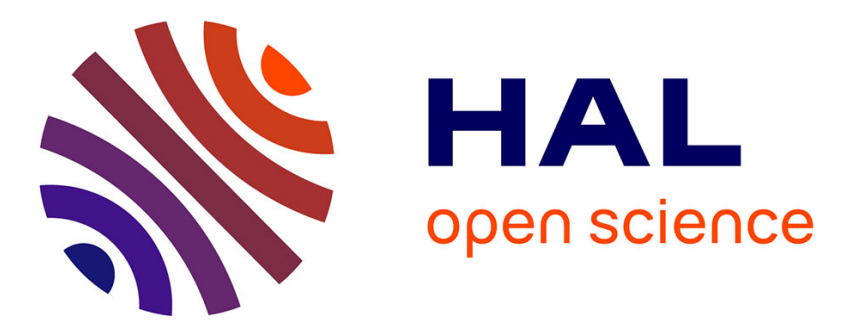

\title{
Local elasticity properties of an amorphous structure: evidences for typical sites and shell structure; dynamic stability
}

\author{
J.C.S. Levy, D. Mercier
}

\section{To cite this version:}

J.C.S. Levy, D. Mercier. Local elasticity properties of an amorphous structure: evidences for typical sites and shell structure; dynamic stability. Journal de Physique, 1984, 45 (2), pp.291-301. 10.1051/jphys:01984004502029100 . jpa-00209755

\section{HAL Id: jpa-00209755 https://hal.science/jpa-00209755}

Submitted on 1 Jan 1984

HAL is a multi-disciplinary open access archive for the deposit and dissemination of scientific research documents, whether they are published or not. The documents may come from teaching and research institutions in France or abroad, or from public or private research centers.
L'archive ouverte pluridisciplinaire HAL, est destinée au dépôt et à la diffusion de documents scientifiques de niveau recherche, publiés ou non, émanant des établissements d'enseignement et de recherche français ou étrangers, des laboratoires publics ou privés. 
Classification

Physics Abstracts

$61.40 \mathrm{D}-64.80 \mathrm{G}-61.10 \mathrm{D}-68.20$

\title{
Local elasticity properties of an amorphous structure : evidences for typical sites and shell structure ; dynamic stability
}

\author{
J. C. S. Levy \\ Laboratoire de Magnétisme des Surfaces, Université Paris 7, 75251 Paris Cedex 05, France
}

and D. Mercier

Laboratoire de Physique des Liquides et Electrochimie (*),

Université Pierre et Marie Curie, 75230 Paris Cedex 05, France

(Reçu le 22 avril 1983, révisé le 30 juin 1983, accepté le 3 octobre 1983)

\begin{abstract}
Résumé. - On définit ici les coefficients élastiques locaux LEC linéaires et quadratiques de structures non périodiques. L'hypothèse d'isotropie macroscopique nous affranchit de distinguer l'orientation des axes de symétrie locaux ce qui limite à 9 le nombre des LEC pertinents. Cette analyse effectuée sur la structure amorphe ML montre l'uniformité de la contrainte hydrostatique $p$ sur chaque couche d'atomes; $p$ prend 5 valeurs distinctes seulement pour les 525 premiers atomes. On définit ainsi 5 catégories de sites et de couches. L'analyse des autres LEC confirme l'existence de ces 5 catégories qui définissent des bandes de valeurs permises pour chacun des LEC. De plus cette analyse donne lieu à une structuration des couches en sous-couches. Les catégories de sites correspondent à des types de symétrie et de topologie locales. L'étude de la dynamique des atomes pour. des modes pratiquement localisés et donc de courte longueur d'onde montre un adoucissement de la fréquence de ces modes sur une couche qui ceinture un domaine d'environ 270 atomes. Ceci définit un seuil de stabilité de la taille de germes. Ces remarques sur la structure en couches, du point de vue de la statique et de la dynamique, nous permettent d'évaluer assez précisément les nombres magiques et les limites de leur évaluation. Les propriétés élastiques globales de la structure ML nue ou dont les interstices sont remplis par de petits atomes, légers, sont calculées grâce au théorème d'additivité. On montre que le rapport $C_{11} / C_{44}$ égal à 2,22 dans les calculs de la structure ML est indépendant de la nature des interactions de paires, tant qu'elles restent à courte portée et favorisent cette structure. Cette mesure est bien en accord avec les résultats expérimentaux. Enfin, un calcul de moyenne locale montre que la taille des fluctuations gelées est d'environ 2 à 3 distances interatomiques. On suggère l'extension de ces résultats à d'autres types d'interaction, comme les interactions molécule-molécule ou nucléon-nucléon.
\end{abstract}

\begin{abstract}
Linear and quadratic Local Elasticity Coefficients (LEC) of non periodic structures are defined here. Assuming a macroscopic isotropy, the directions of local symmetry axes need not be considered, thus only 9 relevant LEC remain. This analysis applied to the ML amorphous structure shows that the local hydrostatic constraint $p$ is uniform on each shell of atoms surrounding the centre of the structure, and takes only 5 different values for the 525 central atoms. Thus $p$ defines 5 categories of sites and shells. The analysis of their other LEC confirms the existence of these 5 categories of shells which define bands of values for each of the LEC. Some internal structuration of the shells, i.e. subbands of LEC, is found. Site categories are analysed in terms of local symmetry and topology. Atom dynamics are considered for possible localized modes with evidence for a softening of the frequencies of the modes more or less localized on a shell which encloses some 270 central atoms. These static and dynamics points of view enable us to make simple remarks on the magic numbers which are usual for shell structures. Global elastic properties such as bulk modulus and sound celerity for the ML structures, bare or filled with small, light atoms are derived, result in a ratio $C_{11} / C_{44}=2.22$, which is independent of the details of a short ranged pair interaction. This ratio is in good agreement with experimental data. Finally the size of frozen fluctuations is shown to be two or three interatomic distances: Applications to different kinds of interactions such as molecule-molecule or nucleonnucleon interactions are suggested.
\end{abstract}

(*) G.R. No 4 du C.N.R.S. 


\section{Introduction.}

Studies of amorphous structures are so numerous that they have to be counted in thousands per year [1]. Moreover the variety of elastic properties of metallic glasses is known to be very great, which is very promising [2]. This is at least one good reason to study the elastic properties of a new theoretically built amorphous structure ML [3], as Srolowitz and Egami [4] did for the model build by Maeda and Takeuchi [5]. In fact, elastic coefficients can be defined from a local analysis in different ways $[4,6]$, and in the following we shall derive an approach which demonstrates additivity theorems and takes advantage of the macroscopic isotropy to restrict the number of relevant Local Elasticity Coefficients (LEC) to 9. The analysis of these local elasticity coefficients can provide an accurate numerical picture [7] of the amorphous structure, because these coefficients measure the local symmetry even if all the distances between neighbours are not exactly the same. In other words, LEC take into account all possible distortions of the amorphous network, and thus, shun the difficult problem of classifying distorted polyhedra into one category or another. Thus the question of existence of different categories of sites becomes a question of statistics and not of direct geometrical observation. However, further geometrical and topological comments can be given to the definition of existing categories. Experimentally, the idea of categories, i.e. of different sites, comes from the measurement of crystalline fields such as hyperfine fields [8] or anisotropy fields. So it could be tempting to derive such a classification not from elasticity coefficients but from crystalline field coefficients. However the connection between interactions responsible for the structure and elastic coefficients is simpler and more direct than that between interactions and local magnetic properties. So it is both easier and more convincing to study the typical sites starting from elastic properties than from magnetic ones. Thus LEC provide a very appreciable amount of information on local structure.

Moreover knowledge of such local elastic coefficients enables us to obtain much information on long wavelength and short wavelength phonons. Furthermore there are many experimental data on both types of phonons and on elastic moduli in amorphous materials $[1,2,9]$, to which our numerical data can be compared. As will be seen from the additivity theorems demonstrated for elastic properties, when considering long wavelength effects and even static behaviour, the local coefficients inside a cell of unit volume must be averaged. This leads to many results, with or without filling of large holes by light atoms, for realistic amorphous structures. On the other hand, LEC can be used to estimate the high frequency phonons by assuming a localization of these modes. Thus the displacement of one atom is sought in the elastic field of the others which remain fixed in this approximation. This assumption enables us to obtain the orders of magnitude of frequencies and especially to see where soft modes can be localized.

Now we are brought back to the very first question, what model structure is to be analysed ? A LEC analysis has been done for the Maeda Takeuchi structure [5] by T. Egami and co-workers [4, 10], but none for the numerous other theoretical structures. As a matter of fact the ML structure [3] has been derived from energetic considerations by means of a variational method [11], and thus has a rather correct density and energy. Usually, other models involve more or less randomization and deserve the name of DRP, dense random packing $[8,12]$. The special interest in the construction of the ML structure lies in its determinism. The analysis of LEC for the ML structure has for goals to show the presence of a chaos and the determination of the nature of this chaos, if any. Chaos is expected in this ML structure because there is a conflict between different structures : the initial germ structure, which is the structure of an icosahedron, cannot propagate in the whole space without conflict, i.e. without frustration. At the very location of these conflicts, there are bifurcations between different structures, and chaos may occur from the large number of these bifurcations. However this chaos is rather specific, it does not involve all the LEC in the same way. Moreover results on DRP simulated structure [4] show such specific chaos which does not involve all the elastic parameters in the same way.

From the analysis of LEC in the ML amorphous structure, many results are obtained. First there are only 5 different hydrostatic constraints $p$ for the 525 first atoms which belong to 14 concentric shells, and all atoms on the same shell experience the same $p$. This is a proof of order along the radial distance, i.e. a proof of shell order. Similar proof of shell order has been observed in DRP constructions [9] with more fluctuations but with the same significance of a basic shell structure : the (RDF) Radial Distribution Functions of compressed or dilated atoms were shifted between them over at least 5 or 6 interatomic distances, which is a very large distance. Thus the shell order which appears in the ML structure, is realistic in DRP structures and is the proof of a partial order well analysed by LEC data. The 5 categories of sites are associated with bands of allowed values of each of the LEC, these bands being distinct for each of the categories and more or less' wide according to the LEC under consideration. In some cases a subshell division can be made, when the bands are divided into independent sub-bands well-defined for several LEC, and especially for $C_{11}$ and $C_{44}$, the main elastic parameters. From this analysis a series of shells and subshells can be defined and we thus can introduce a list of magic numbers which define the completion of successive shells and subshells. These magic numbers are compared with those obtained for clusters of rare gas atoms [13], metallic atoms [14], or molecules [15]. A comparison may even be risked with 
clusters of nuclear matter because of the generality of the short-ranged potential here introduced; the shell model is known to be efficient for nuclear matter [16], appears as well for the ML structure, and the dynamic effect described by quadratic LEC such as $C_{11}$ and $C_{44}$ shows a weak stability for clusters of about 270 particles.

Knowledge of the LEC enables us to estimate the frequency of short wavelength modes and to show where localized modes can occur. Such localized modes are obtained on a shell which encloses some 270 atoms. On this shell the local symmetry is oblong. The detailed motion of such localized modes may be a displacement of the shell only such as a rotation, or a rotation of the whole internal sphere without any other external motion. This mode, called a roton mode, was predicted by Landau for liquids a long time ago [17] and Heritier et al. proposed a similar mode, for amorphous materials [18], which was called solidon because of the rigid central unit. Such a connection between localized modes and local topology was recently proposed by Kleman [19]. Hence, one question is raised. If the structure is restricted to this shell and its internal particles, we have a cluster of about 300 particles, which is still metastable statically because our variational arguments of reference 3 are still valid. But the LEC's of this cluster are expected to have a smaller value than those of the ML structure because of the relative lack of neighbors for peripheral particles. Hence the poor dynamic stability of such shells in the ML structure is aggravated for these 300 particles clusters. So this size of about 300 particles is expected to be critical. This remark can be compared to the observation of a maximum size of about 300 molecules for clusters of various molecules $[14,15]$. Moreover this size of about 300 particles is that of the nucleus of actinide elements, which is a well known critical size. As nucleons interact mainly via a short-range potential, the Yukawa potential [16], this comparison even if rough is a correct first approximation. Moreover the tunnelling character of natural radioactivity corresponds well to the glassy state and its numerous tunnelling effects.

In the present work these problems of dynamic stability are not solved but only introduced. But already they show their generality and their common. aspects, on the basis of a pair potential or pseudopotential approach. And the introduction of the dynamic instability made above raises the problem of the stability of realistic amorphous materials. Either the stability of the ML amorphous structure is restricted to the domain of very low temperatures, or the ML structure must be stuffed with small atoms in the big holes in such a way that atomic motions during soft mode propagation will be quite small and tunnelling effects strongly forbidden. Giving this, the additivity theorem enables us to calculate the elastic macroscopic properties from the LEC. They are compared to experimental data with a very good agreement on the ratio of the averaged values of $C_{11}$ and $C_{44}$ [20]. This ratio does not depend on the detailed interaction as long as it is still short ranged. Thus the microstructure depicted by the ML structure, has macroscopic consequences which correspond to the existing date. In order to make this result easily understandable, it can be said that the ML structure simply solves the conflict between a local stable structure, i.e. the icosahedron and its spatial propagation which cannot occur without frustration [21]. And the ratio $C_{11} / C_{44}$ depends less on the solution of the frustration problem than on the local structure of the unfrustrated part.

In section 1 will be defined the linear coefficients which are typical of the low symmetry of amorphous structures, then the quadratic ones, i.e. the stiffness coefficients and the additivity rule will be derived.

Section 2 is devoted to the results for ML structure : shell model, classification of the sites, size of fluctuations.

The generality of the shell model, elastic parameters and detailed considerations of normal modes will be shown in section 3 .

\section{Local elastic coefficients LEC : definitions.}

The Lennard-Jones potential $U$ used as pair potential is :

$$
U_{(r)}=-2 r^{-6}+r^{-12}
$$

where $r$ is the pair distance.

With such a potential the ML structure predicted in reference 3 is stable when using suitable boundary conditions, i.e. fixed atoms at the periphery. In the following, literal equations suppose only a radial pair potential, i.e. $U$ depends only on $|\mathbf{r}|$. Local phenomena are considered, thus one central atom $O$ can be taken as fixed and the vectors which define its neighbours will be called $A_{i}$. A local deformation defines the strain tensor $\varepsilon$ by :

$$
\mathbf{A}_{i}^{\prime}=(1+\varepsilon) \mathbf{A}_{i}
$$

where $A_{i}^{\prime}$ is the new vector. Classically $[4,6]$, one defines a 6 dimension strain vector $\varepsilon$ by :

$$
\begin{aligned}
& \varepsilon_{1}=2 \varepsilon_{x x}, \quad \varepsilon_{2}=2 \varepsilon_{y y}, \\
& \varepsilon_{3}=2 \varepsilon_{z z}, \quad \varepsilon_{4}=\varepsilon_{y z}+\varepsilon_{z y} \\
& \varepsilon_{5}=\varepsilon_{x z}+\varepsilon_{z x} \text { and } \varepsilon_{6}=\varepsilon_{y x}+\varepsilon_{x y} \text {. }
\end{aligned}
$$

Thus the variation of $r_{i}=A_{i}$ in the deformation can be analysed in two parts of increasing order in powers of :

$$
\mathrm{d} r_{i}=\mathrm{d} r_{i}^{(1)}+\mathrm{d} r_{i}^{(2)}
$$

where higher terms are neglected. With $\alpha, \beta$ and $\gamma$ as coordinates of $\mathbf{A}$, the corrective terms are :

$$
r \mathrm{~d} r^{(1)}=\overline{\alpha^{2}: \beta^{2}: \gamma^{2}: \beta \gamma \vdots \gamma \alpha \vdots \alpha \beta} \text {.(ع) }
$$

where $\varepsilon$ is a column vector. 
And

$$
2 r \mathrm{~d} r^{(2)}=\varepsilon^{\mathrm{t}} \cdot\left[\begin{array}{cccccc}
\alpha^{2} & 0 & 0 & 0 & \alpha \gamma & \alpha \beta \\
0 & \beta^{2} & 0 & \beta \gamma & 0 & \alpha \beta \\
0 & 0 & \gamma^{2} & \beta \gamma & \alpha \gamma & 0 \\
0 & \beta \gamma & \beta \gamma & \beta^{2}+\gamma^{2} & \alpha \beta & \alpha \gamma \\
\alpha \gamma & 0 & \alpha \gamma & \alpha \beta & \alpha^{2}+\gamma^{2} & \beta \gamma \\
\alpha \beta & \alpha \beta & 0 & \alpha \gamma & \beta \gamma & \alpha^{2}+\beta^{2}
\end{array}\right] .(\varepsilon)
$$

where $\varepsilon^{t}$ is the transposed row vector.

The variation $\Delta U_{i}$ of the potential, due to the deformation can be written (up to terms of second order in $\varepsilon$ ) :.

$$
\Delta U_{i}=\mathrm{d} r_{i}^{(1)} \frac{\partial U_{i}}{\partial r}+\mathrm{d} r_{i}^{(2)} \frac{\partial U_{i}}{\partial r}+\frac{1}{2}\left(\mathrm{~d} r_{i}^{(1)}\right)^{2} \frac{\partial^{2} U_{i}}{\partial r^{2}}
$$

This can be translated into

$$
\Delta U_{i}=\mathcal{A}_{i} \varepsilon+\varepsilon^{\mathrm{t}} \mathcal{B}_{i} \varepsilon+\varepsilon^{\mathrm{t}} \mathcal{C}_{i} \varepsilon
$$

with an obvious notation which enables us to sum over all the neighbours $i$ of the central atom $O$ where local elastic coefficients LEC are to be defined.

$$
\sum_{i} \mathcal{A}_{i}=\mathcal{A}, \quad \sum_{i} \mathfrak{B}_{i}=\mathfrak{B} \text { and } \sum_{i} \mathcal{C}_{i}=\mathcal{C}
$$

Matrices $\mathcal{A}, \mathcal{B}$ and $\mathcal{C}$ are easily deduced from equations 5 and 6 :

$\mathcal{A}=\sum \frac{\partial U_{i}}{\partial r} \times \frac{1}{r_{i}} \times \overline{\alpha_{i}^{2}: \beta_{i}^{2} \vdots \gamma_{i}^{2} \vdots \beta_{i} \gamma_{i}: \alpha_{i} \gamma_{i} \vdots \alpha_{i} \beta_{i}}$

$\mathcal{B}=\sum \frac{\partial U_{i}}{\partial r_{i}} \times \frac{1}{2 r_{i}} \times\left[\begin{array}{cccccc}\alpha_{i}^{2} & 0 & 0 & 0 & \alpha_{i} \gamma_{i} & \alpha_{i} \beta_{i} \\ 0 & \beta_{i}^{2} & 0 & \beta_{i} \gamma_{i} & 0 & \alpha_{i} \beta_{i} \\ 0 & 0 & \gamma_{i}^{2} & \beta_{i} \gamma_{i} & \alpha_{i} \gamma_{i} & 0 \\ 0 & \beta_{i} \gamma_{i} & \beta_{i} \gamma_{i} & \beta_{i}^{2}+\gamma_{i}^{2} & \alpha_{i} \beta_{i} & \alpha_{i} \gamma_{i} \\ \alpha_{i} \gamma_{i} & 0 & \alpha_{i} \gamma_{i} & \alpha_{i} \beta_{i} & \alpha_{i}^{2}+\gamma_{i}^{2} & \beta_{i} \gamma_{i} \\ \alpha_{i} \beta_{i} & \alpha_{i} \beta_{i} & 0 & \alpha_{i} \gamma_{i} & \beta_{i} \gamma_{i} & \alpha_{i}^{2}+\beta_{i}^{2}\end{array}\right]$

$\mathcal{C}=\sum \frac{1}{2 r_{i}^{2}} \frac{\partial^{2} U_{i}}{\partial r_{i}^{2}} \times\left[\begin{array}{cccccc}\alpha_{i}^{4} & \alpha_{i}^{2} \beta_{i}^{2} & \alpha_{i}^{2} \gamma_{i}^{2} & \alpha_{i}^{2} \beta_{i} \gamma_{i} & \alpha_{i}^{3} \gamma_{i} & \alpha_{i}^{3} \beta_{i} \\ \alpha_{i}^{2} \beta_{i}^{2} & \beta_{i}^{4} & \beta_{i}^{2} \gamma_{i}^{2} & \beta_{i}^{3} \gamma_{i} & \alpha_{i} \beta_{i}^{2} \gamma_{i} & \alpha_{i} \beta_{i}^{3} \\ \alpha_{i}^{2} \gamma_{i}^{2} & \beta_{i}^{2} \gamma_{i}^{2} & \gamma_{i}^{4} & \beta_{i} \gamma_{i}^{3} & \alpha_{i} \gamma_{i}^{3} & \alpha_{i} \beta_{i} \gamma_{i}^{2} \\ \alpha_{i}^{2} \beta_{i} \gamma_{i} & \beta_{i}^{3} \gamma_{i} & \beta_{i} \gamma_{i}^{3} & \beta_{i}^{2} \gamma_{i}^{2} & \alpha_{i} \beta_{i} \gamma_{i}^{2} & \alpha_{i} \beta_{i}^{2} \gamma_{i} \\ \alpha_{i}^{3} \gamma_{i} & \alpha_{i} \beta_{i}^{2} \gamma_{i} & \alpha_{i} \gamma_{i}^{2} & \alpha_{i} \beta_{i} \gamma_{i}^{2} & \alpha_{i}^{2} \gamma_{i}^{2} & \alpha_{i}^{2} \beta_{i} \gamma_{i} \\ \alpha_{i}^{3} \beta_{i} & \alpha_{i} \beta_{i}^{3} & \alpha_{i} \beta_{i} \gamma_{i}^{2} & \alpha_{i} \beta_{i}^{2} \gamma_{i} & \alpha^{2} \beta_{i} \gamma_{i} & \alpha_{i}^{2} \beta_{i}^{2}\end{array}\right]$

In the matrices $\mathcal{A}$ and $\mathscr{B}$ there are only six independent quantities which are nil if all the $r_{i}$ 's are equal to $r_{0}$ where $U(r)$ is minimum. In ML structure numerous interatomic distances show a contraction : $r<r_{0}$ and so $U^{\prime}<0$, while some ones show a dilation $r>r_{0}$ and $U^{\prime}>0$. The stability of the structure implies more contractions than dilations and this will be confirmed in the numerical analysis, by the negative sign of the 3 first coefficients of $A$.

The symmetries of matrix $\mathcal{C}$ are wellknown [6] and give rise to the 6 Cauchy relations, thus there are only 15 independent parameters involved in $\mathcal{C}$. When the total local energy $U$ is derived as a function of the strain, the stress $\mathcal{F}$ is obtained :

$$
\mathfrak{F}_{k}=\frac{\partial U}{\partial \varepsilon_{k}}=\mathcal{A}_{k}+2[(\Re+\mathcal{C}) \varepsilon]_{k}
$$

where $k$ labels the $k$ th column of the matrix in these covariant notations. The following comment is 
obvious : $\mathcal{A}_{\boldsymbol{k}}$ is the stress due to frozen fluctuations while $(\mathfrak{B}+\mathcal{C})$ is the matrix of stiffness coefficients. $\mathcal{C}$ looks like the classical stiffness matrix, thus $\mathcal{B}$ is the correction due to frozen fluctuations.

There are two additivity rules for elastic coefficients. First, there is an additivity rule for local elastic coefficients if the holes are filled with small atoms, or if there is a vacancy. In both cases the change in LEC for other sites results from the uniformity of the strain $\varepsilon$ in elasticity theory and the additivity of the two body interactions. Thus LEC are additive with respect to atoms filling an interstitial site or leaving a vacancy, under the assumption of no rearrangement, which is a first approximation. The second additivity rule deals with the change from local elasticity coefficients to global elastic coefficients. Now are added the energies $\Delta U_{i}$ for all the particles $i$ which belong to the same unit volume. This additivity is simple for pair potentials and leads to add the matrices $A_{i j}, B_{i j}, C_{i j}$, over both the indices $i$ and $j$, because the strain vector $\varepsilon$ is always uniform. The forces on the unit volume, i.e. the stresses, are derived from an obvious generalization of equation 13 , where $\varepsilon$, which is the local strain vector in this $6 \mathrm{~d}$ linear space, is also the global strain vector because of the central assumption of uniform strains in elasticity theory. Thus elastic coefficients result from the sum of LEC, for sites belonging to a unit volume. Statistically an isotropic averaging of these elastic coefficients can be expected, while in crystals, symmetry rules infer direct conditions on the LEC.

\section{LEC numerical results.}

Each site is represented by 21 coefficients : 6 for the matrices $A$ and $B$ and 15 for the stiffness matrix $C$. A systematic study of these coefficients involves many figures and simplification is useful. One can note from the tensorial nature of $A$ and $C$ that these 21 LEC involve the definition of different local symmetry axes. These local symmetry axes vary from site to site, and following the classical observation of random anisotropy [21], one can easily admit that these directions are randomly distributed on any macroscopic scale. This remark enables us to leave out this directional character and thus 9 relevant parameters remain. The careful observation of direction distribution will be described in an other paper.

The choice of ML structure is due to its deterministic nature, as already said in the Introduction, and thus the LEC's will show the nature and the values of any disorder in such a deterministic structure. It should be recalled that the ML structure is obtained from a variational method which leads to the equation of structural propagation [11] :

$$
n_{k} V_{k}=0
$$

where $n_{k}$ is the Fourier transform of the density while $V_{k}$ is the Fourier transform of the pair potential.
This equation of structural propagation is integrated [11]:

$$
n(\mathbf{r})=\sum c_{j} \exp \left[i \mathbf{k}_{j} \cdot \mathbf{r}\right]
$$

where the $\mathbf{k}_{j}$ 's are the nodes of $V_{k}$. The $c_{j}$ 's are determined from boundary conditions, i.e. commensuration with an icosahedral germ and $3 \mathrm{~d}$ propagation [3, $11,21]$. The resulting density $n(\mathbf{r})$ is a continuous function of which the peaks define the ML network. Naturally we exclude too close peaks by selecting the highest one. This kind of conflict is responsible for bifurcations and, finally chaos, because any choice leads to a series of subsequent choices [21].

A preliminary study of LEC on ML structure has been carried out [7]. It emphasizes the central symmetry of the ML structure which enables us to classify the particles according to their distance $R$ from the centre. One can count them in this way and classify each of them by an unambiguous number $N . N=1$ means the centre, $N=2$ the nearest neighbour of the centre, and so on. This defines two ways of classifying the particles, either along $R$ or along $N^{1 / 3}$ as will be used in the following figures. The classification according to $R$, i.e. the radial distance, emphasizes the shell structure, while the other classification emphasizes the distribution of LEC.

In the practical calculations, we account only the interaction between nearest neighbours and thus assume a strong screening of the long range interaction. This approximation does not perturb the principal significance of the results. Computations were carried out on IRIS 80, a CII Honeywell Bull computer at the Paris, Jussieu University Centre.

2.1 Shell STRUCTURE AND LiNEAR LEC. - By linear LEC it is meant the 6 coefficients of matrices $A$ and $\mathcal{B}$, which reduce to $3: p, \tau$ and $p^{\prime}$ when the directional character of the main symmetry axis which is randomly varied, is omitted.

$$
\begin{aligned}
p & =\frac{\mathcal{A}_{1}+\mathcal{A}_{2}+\mathcal{A}_{3}}{3} \\
\tau & =\sqrt{\frac{\left(\mathcal{A}_{1}-\mathcal{A}_{2}\right)^{2}+\left(\mathcal{A}_{2}-\mathcal{A}_{3}\right)^{2}+\left(\mathcal{A}_{3}-\mathcal{A}_{1}\right)^{2}}{3}} \\
p^{\prime} & =\sqrt{\frac{\mathcal{A}_{4}^{2}+\mathcal{A}_{5}^{2}+\mathcal{A}_{6}^{2}}{3}}
\end{aligned}
$$

$p$ is the hydrostatic pressure of Srolovitz and Egami [4], $\tau$ their shear stress. As already stated $p$ is the negative measure of the contraction of the particle, while $\tau$ measures the anisotropy of the contraction-dilatation. Figure 1 shows $p(R)$ for the 500 central atoms : with strong evidence for a shell structure and 5 values of the local pressure with different contractions of the considered sites. Figure 2 shows $p\left(N^{1 / 3}\right)$ with the same evidence which is now weighted by the number of 
$10^{1}$

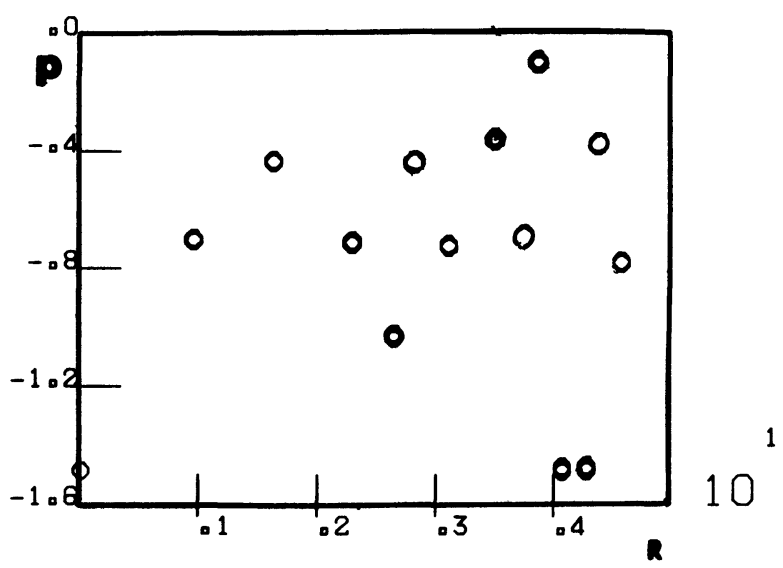

Fig. 1. - Local hydrostatic pressure $p$ as a function of the radial distance $R$ for the 525 central atoms of the ML structure. 14 shells with about 5 different values of $p$ can be noticed, i.e. five typical sites. $p$ is also called " constraint" throughout this paper.

particles in the shell. Figures 3 and 4 show $\tau\left(N^{1 / 3}\right)$ and $p^{\prime}\left(N^{1 / 3}\right)$. They define the isotropic shells, with $\tau$ and $p^{\prime}$ nearly equal to 0 , and measure the intensity of anisotropy of the others shells, because $\tau$ measures the intensity of anisotropy of diagonal terms while $p^{\prime}$ measures the intensity of off-diagonal terms. Numerous shells are structured into subshells with different subbands of values of $\tau$ and $p^{\prime}$. On the other hand one can see the large scale of variations of the parameters $\tau$ and $p^{\prime}$ which confirms the amorphous nature of the ML structure.

2.2 QUADRATIC LEC : STIFFNESS COEFFICIENTS SITES. - The matrix elements of $\mathcal{B}$ are in fact much lower than these of $\mathcal{C}$, which means the frozen fluctuations do not strongly alter the stiffness coefficients, as can be expected from well relaxed amorphous samples. We take advantage of this feature by computing only the matrix elements of $\mathrm{C}$. Always leaving out the direc-

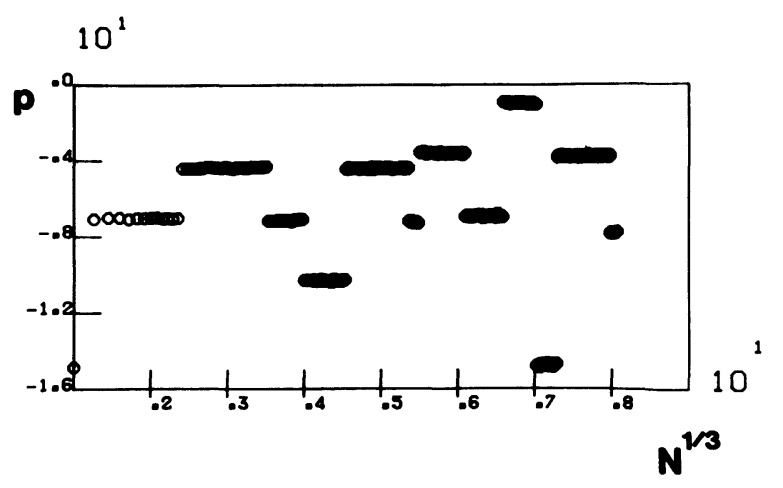

Fig. 2. - Local hydrostatic pressure $p$ as a function of $N^{1 / 3}$ for the 525 central atoms of the ML structure.

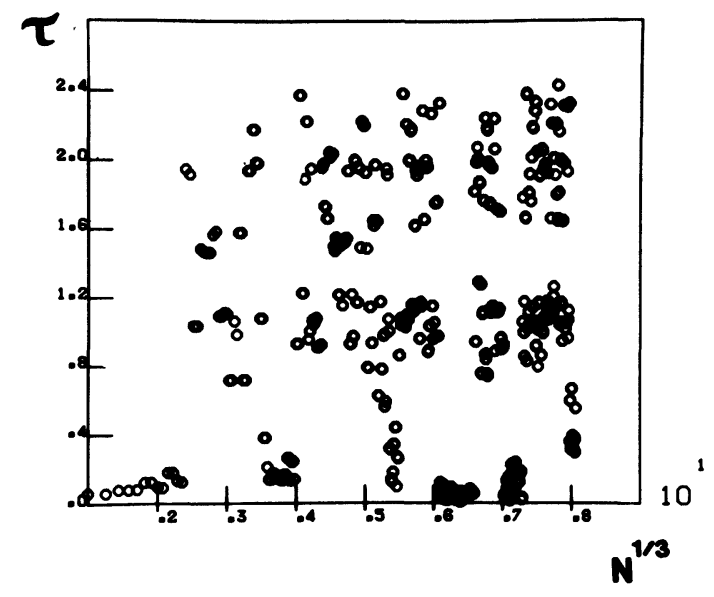

Fig. 3. - Local shear stress $\tau$ as a function of $N^{1 / 3}$. There is a large distribution with isotropical sites $(\tau \sim 0)$ and anisotropical sites. Different sublayers of anisotropical shells can be distinguished : $\tau<1.2$ or $\tau>1.2$. The separation is fairly clear.

tional character which can be expected to be randomly distributed as for the anisotropy direction of amorphous materials [22], several relevant coefficients remain :

$$
\begin{aligned}
& C_{11}=\frac{\mathrm{C}_{11}+\mathrm{C}_{22}+\mathrm{C}_{33}}{3} \\
& C_{44}=\frac{\mathfrak{C}_{44}+\mathfrak{C}_{55}+\mathfrak{C}_{66}}{3} \\
& C_{11}^{\prime}=\sqrt{\frac{\left(\mathrm{C}_{11}-\mathrm{C}_{22}\right)^{2}+\left(\mathrm{C}_{22}-\mathrm{C}_{33}\right)^{2}+\left(\mathrm{C}_{33}-\mathrm{C}_{11}\right)^{2}}{3}} \\
& C^{\prime}=\sqrt{\frac{\mathcal{C}_{15}^{2}+\mathcal{C}_{16}^{2}+\mathcal{C}_{24}^{2}+\mathcal{C}_{26}^{2}+\mathcal{C}_{34}^{2}+\mathcal{C}_{35}^{2}}{3}} \\
& C_{44}^{\prime}=\sqrt{\frac{\left(\mathrm{C}_{44}-\mathrm{C}_{55}\right)^{2}+\left(\mathrm{C}_{55}-\mathrm{C}_{66}\right)^{2}+\left(\mathrm{C}_{66}-\mathrm{C}_{44}\right)^{2}}{3}} \\
& c^{\prime}=\sqrt{\frac{\mathrm{C}_{14}^{2}+\mathrm{C}_{25}^{2}+\mathfrak{C}_{36}^{2}}{3}} .
\end{aligned}
$$




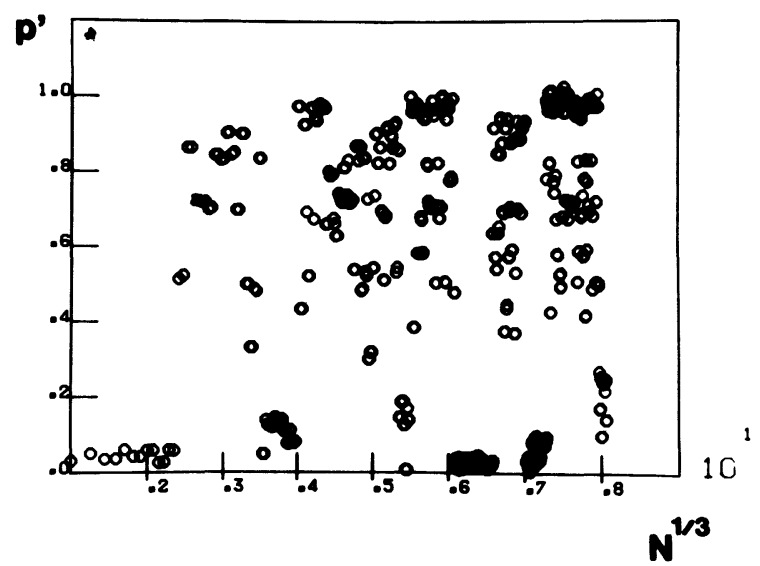

Fig. 4. - Local constraint $p^{\prime}$ as a function of $N^{1 / 3}$. The already defined isotropic shells have a $p^{\prime}$ nil, while for the other shells $p^{\prime}$ is widely distributed from 0 to 1.0 .

In this classification $C_{11}$ and $C_{44}$ resemble the usual parameters [6], with here an obvious symmetrization, which forbids the definition of $C_{12}$. Figures 5,6 and 7 show respectively $C_{11}, C_{44}$ and $C_{11}^{\prime}$ as a function of $N^{1 / 3}$. The order of parameters $C_{11}$ to $c^{\prime}$ listed in equations 17 is that of decreasing magnitude from $C_{11}$ to $c^{\prime}$, which confirms the rather strong spherical symmetry on each site, because $C_{11}^{\prime}, C^{\prime}, C_{44}^{\prime}$ and $c^{\prime}$ measure different deviations from this symmetry. These results can be compared with those for the shell assumption as in table $\mathrm{I}$, where the values of $p, \tau, p^{\prime}, C_{11}, C_{44}$ and so on, are noted for the different shells. The conver-

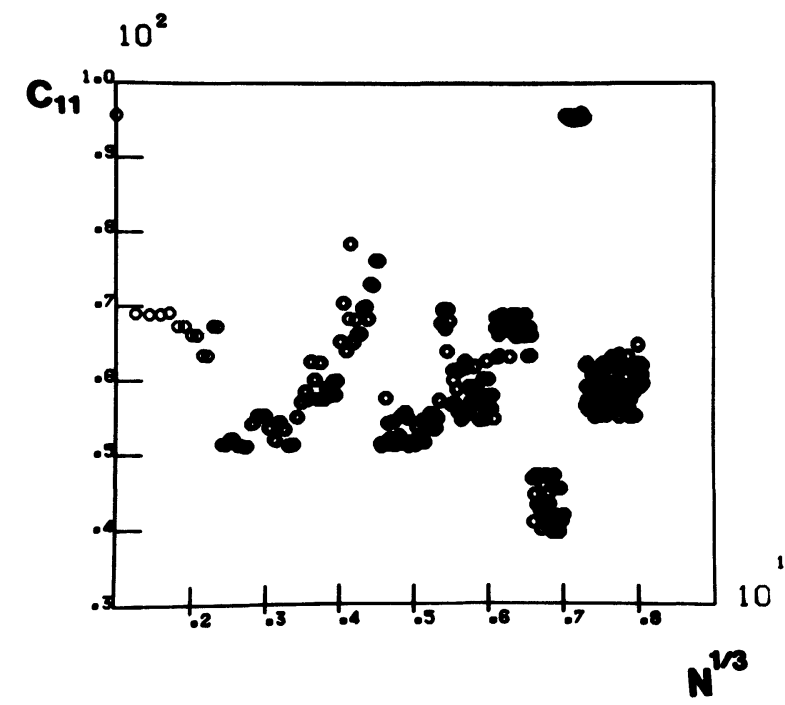

Fig. 5. - Local stiffness constant $C_{11}$ as a function of $N^{1 / 3}$. The following shells appear : the isotropic ones with a uniform value, the anisotropic ones with a rather wide distribution of values. The sublayers defined on figure 3 are clearly seen, for instance very large $\tau$ values are correlated with small $C_{11}$ value especially for the second shell where this remark is used to select a stable layer. For $N^{1 / 3}$ near 6.8 there is a catastrophe in the $C_{11}$ values which are very low. This is correlated with a low value of $p$. It is a critical size for small clusters which are badly elastically coupled to others.

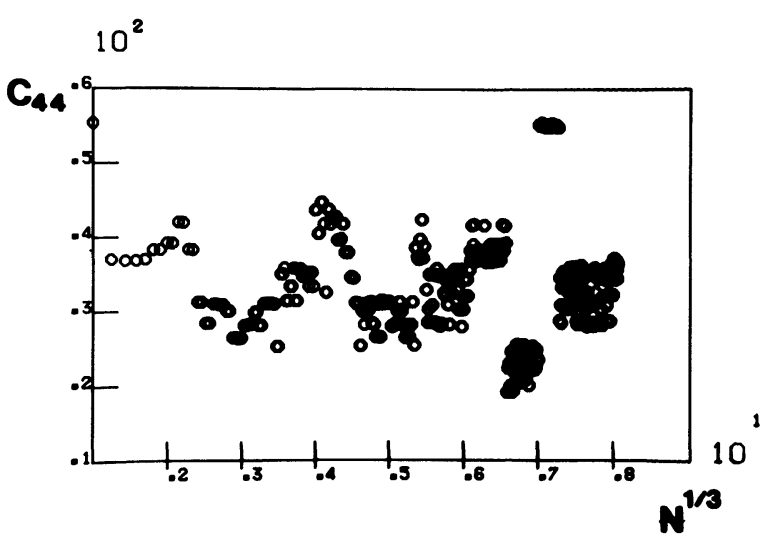

Fig. 6. - Local transverse stiffness coefficient $C_{44}$ as a function of $N^{1 / 3}$. Shells and subshells appear. The catastrophe at $N^{1 / 3}=6.8$ is less strong than for $C_{11}$.

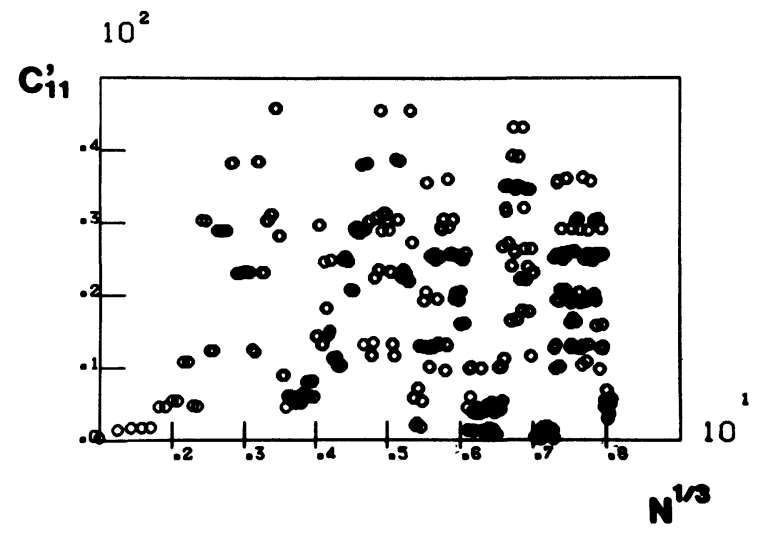

Fig. 7. - Local stiffness coefficient $C_{11}^{\prime}$ as a function of $N^{1 / 3}$. This coefficient sensitive to the anisotropy clearly shows the catastrophe at $N^{1 / 3}=6.8$.

gence of the different data for the evidence of shells and of a few typical sites should be noted. There are two general categories of sites, the isotropic ones which have a correct coordination number $N_{\mathrm{c}}$ and are under hydrostatic pressure, and the anisotropic ones which have a lower $N_{\mathrm{C}}$ and thus are frustrated. Inside these categories, there is a further classification into several classes : A : isotropic, D : very isotropic, B : moderately anisotropic, $\mathrm{C}$ : anisotropic, $\mathrm{E}$ : oblong and very anisotropic. In other words sites $A$ and $D$ are unfrustrated while sites $\mathrm{B}, \mathrm{C}$ and $\mathrm{E}$ are more or less frustrated. Figures 1 and 2 show the natural order of successive shells, where the pressure oscillations correspond to the alternance of frustrated and unfrustrated shells. Figures 3 to 7 demonstrate the kind of chaos which observed for the different LEC but there is no one-toone correspondence. Each shell defines a band of values for each LEC, and mixing of shells is rare : even the shell $D^{\prime}$ which is rather particular has a « $D$ class ». There is a chaos which seems typical of amorphous structures, because of the partial order which can be expected in this 4th state of matter, and because of the comparison with other results on models [4]. 
Table I. - L.E.C. for different shells classification and name of the shells according to N.

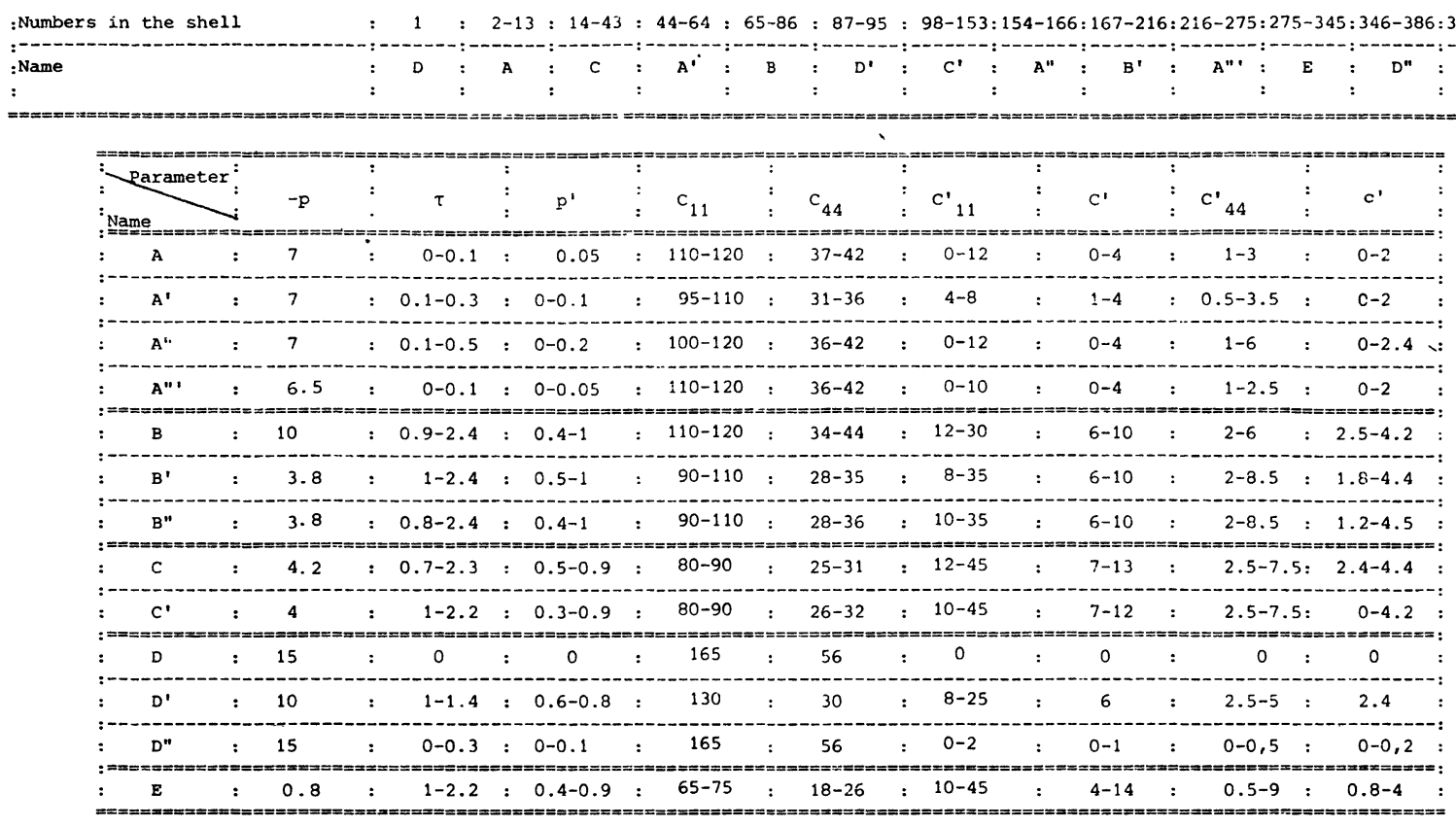

The local stiffness coefficients can be used to define approximate local modes, as global stiffness coefficients define long wavelength modes [23]. For these approximate local modes the $N$ th atom or particle is mobile while the others are assumed to be fixed. Two typical Einstein modes are defined by $C_{11}$ and $C_{44}$. $C_{11}$ is the spring constant of a rectilinear motion where displacements and forces are parallel, while $C_{44}$ is the spring constant of a circular motion where displacements and forces are orthogonal. These motions are the local translation of longitudinal and transverse phonon modes [23]. Of course soft modes can be observed when $C_{11}$ or $C_{44}$ tends towards 0 . This occurs for $N^{1 / 3} \sim 6.5$, i.e. $N \sim 276$ for the shell called E, i.e. with oblong symmetry.

The ratio of softening, i.e. $\left(C_{\mathrm{E}} / C_{\mathrm{A}}\right)$ which compares the two classes of sites is nearly 0.5 , as can be seen on figures 5 and 6 for $C_{11}$ and $C_{44}$ and in table $\mathrm{I}$. This is real softening. As already said, if the size of the previous clusters is restricted in order to have only 300 particles with the same ML structure which, because of the variational principle, is still a fairly stable cluster, the additivity theorem 1 shows that there is a decrease of LEC at the external shell; thus the ratio of softening $\left(C_{\mathrm{E}} / C_{\mathrm{A}}\right)$ here becomes lower than 0.5 . The dynamic stability of such a cluster then becomes a problem. Comparison with nuclear matter, i.e. the radioactivity catastrophe for actinides, suggests itself. There is the instability of pentagonal structures [13] too. Of course this is not a demonstration but a mere connection which can be improved by further analysis. When speaking of a comparison with finite cluster, one has to introduce the "magic numbers » [15]. These magic numbers have been defined for the shell model. But the present discussion reveals the value of an analysis of dynamics, i.e. of the dynamic stability which has to be checked for each shell.

The first shell, $\mathrm{D}$, is complete with $N=1$. The second shell, A, is fairly homogeneous and is complete when $N=13$, which is the second magic number. The third shell, C, is complete for $N=43$, but is not homogeneous, especially for $C_{11}: 16$ particles have a $C_{11}$ equal to 80 . Thus one can admit the low stability of these particles for a real small cluster and then assume as a true magic number : $43-16=$ 27. This discussion shows the accuracy of the definition of these numbers to a few units. The next shell $\mathbf{A}^{\prime}$ contains the 20 particles $N=44$ to $N=63$. Thus the new magic number would be $27+20=47$ if the 16 atoms of the previous shell are not stabilized by the existence of the new shell. If the previous subshell of $\mathrm{C}$ is more or less filled when this shell $\mathrm{A}^{\prime}$ is filled, the realistic magic number lies in the range 47-63. The 55-atom cluster of Farges [13] corresponds to this range, and 55 is also a well-known magic number for clusters of atoms [13] or molecules [14, 15]. Such as for nuclear matter where the abundance of $\mathrm{Fe}$ and $\mathrm{Ni}$ is associated with this magic number of nucleons. The next shell, B, contains 26 atoms. Thus reasonably the next magic number lies between 81 and 89 , because this shell is rather stable. The $\mathrm{D}^{\prime}$ shell which is quite stable follows with a magic number of 91 to 99 . Then the next shell, $C^{\prime}$, will lead to a magic number 147 to 155 , but as already observed for the $C$ shell, there is a weak dynamic stability of one subshell, the $C_{11}$ 's go down to values of 80 for the extended cluster. This low stability is associated with the nucleon numbers of rare earths, and probably explains their rarity. 
2.3 Mean values and Size OF THE Fluctuations. From the second additivity rule, mean values are important to know and easy to obtain. It is more difficult to estimate that of the typical size of the frozen fluctuations which can be thought of as a superstructure such as that observed in some theoretical structures [5]. A first analysis consists in averaging the LEC over the nearest neighbour sites. This has been done for all the parameters which gave as a result a very short fluctuation length as can be seen on figure 8 . Figure 8 shows the averaged values $V p, V \tau$ and $V C_{11}$ of $p, \tau$ and $C_{11}$ respectively, which are averaged over nearest neighbouring sites, as a function of $N^{1 / 3}$.

The efficiency of the averaging can be seen even if the soft mode catastrophe remains apparent. Practically this means that the wavelength of the frozen fluctuations, i.e. of the superstructure, is about two or three interatomic distances, with an alternance of frustrated layers and unfrustrated layers, or shells. By frustration we mean lack of neighbours, anisotropy and so on, while unfrustration means isotropy, high level of coordination, hydrostatic pressure and so on.

\section{Further analysis of the results.}

3.1 OTHER EVIDENCES FOR A SHELL STRUCTURE. First of all it can be noted that though the ML structure is easily seen to have a shell structure, ML is not the only one. For instance Srolovitz and Egami [10] noticed that in the MT structure [5] the averages of radial distribution functions taken at sites of low or high hydrostatic pressure, are similarly distributed over distances of several atomic distances. This means a rather regular structure centred over contracted or dilated atoms with no rapid damping of the shell structure. So this is a strong numerical proof for the shell structure of this MT model.

The present theory works with amorphous materials, i.e. large clusters, and its application to small clusters where boundary problems occur, is far from perfect. But it is not quite unfit, at least when $N>13$. This explains the agreement of magic numbers with experimental work on metal clusters [14] or molecule clusters [15]. The next step is to go from the LennardJones potential to nuclear interactions, which leads to the well known shell models [16] of the nucleus. As nucleons are fermions they experiment a strong repulsion at short distances while Yukawa potential is attractive at larger distances and very strongly screened. So the effective pseudopotential of nuclear forces does not have a shape very different from that of the Lennard-Jones potential. This explains the similarity of the results even if many details are neglected.

3.2 Elastic Parameters. - One has here a calculation of the averaged elastic parameters which are those of an isotropic sample, with $C_{12}=C_{44}$ because of the Cauchy relations.
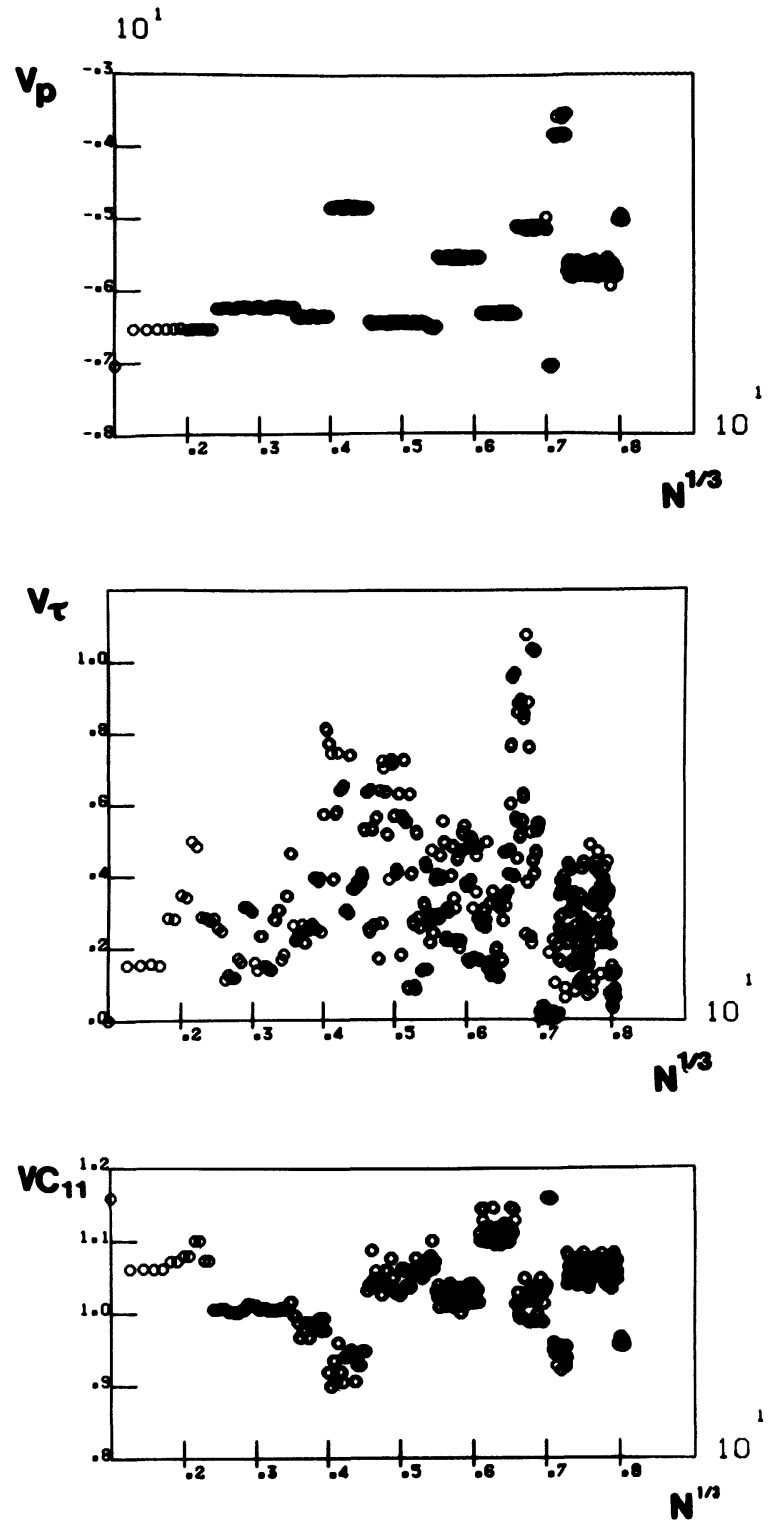

Fig. 8. - The average values $V p, V \tau$ and $V C_{11}$ of $p, \tau$ and $C_{11}$ as a function of $N^{1 / 3}$; The averages have been taken over the nearest neighbours of each site $(d<1.3)$. The amplitude of variation is shown to exhibit a strong decrease compared with that of the non averaged values given on curves 2,3 and 5 . The $N^{1 / 3}=6.8$ catastrophe is damped but still evident.

$$
\begin{aligned}
& C_{11} \# 80 \mathrm{u} \\
& C_{44} \# 36 \mathrm{u}
\end{aligned}
$$

and the bulk modulus

$$
B \# 50 \mathrm{u}
$$

where the unit $u$ is the size of the potential well divided by the cube of the atomic distance, according to the definition of $U$ by equation 1 . The ratio $C_{11} / C_{44}=$ 2.22 is dimensionless and thus has a universal character for this type of structure and for the interaction which is strongly restricted to first neighbours. This 
ratio measures the ratio of sound celerities for longitudinal and transverse vibrations and has been experimentally measured by Bellessa and Bethoux [20] to be nearly 2.22 at $0 \mathrm{~K}$ in $\mathrm{Pd}_{80} \mathrm{Si}_{20}, \mathrm{Ni}_{80} \mathrm{P}_{20}$ and $\mathrm{Co}_{80} \mathrm{P}_{20}$ in very good agreement with the present work. It has to be said that the experimental work showed a linear variation of $C_{11}$ and $C_{44}$ with temperature which is evidence for low lying excited states. These low lying excited states are not in contradiction with the ML structure [3], but their contribution is out of the scope of the present paper.

This experimental agreement is not due to chance, and can be explained in more detail. First the ML structure contains many large holes, about $20 \%$ of the number of particle if the lowest hole radius is taken as 0.6 times the atomic radius [3,7]. Thus the ML structure contains many large holes, about $20 \%$ of the amorphous alloys where $\mathbf{M}$ is a large metallic atom and $\mathrm{m}$ a small metalloid atom. Similar remarks have been made by Polk for other models with the same density of large holes [24]. Then assuming the ML structure stuffed with metalloid atoms for the structure of amorphous samples studied by Bellessa et al. [20], one can derive the elastic constants from the additivity rules. The main part comes from the $80 \%$ of metallic atoms or ions which are the more numerous and the heaviest. At the acoustic low frequencies light metalloid atoms follow instantaneously the heavy metallic atoms, and thus their contribution to LEC is weaker than their atomic density. Moreover this stuffing of holes leads to a homogenization of LEC throughout, and especially for the $\mathrm{E}$ shell. Thus the changes in $C_{11}$ and $C_{44}$ due to this stuffing will be nearly proportional to $C_{11}(\mathrm{~A})-C_{11}(\mathrm{E})$ and $C_{44}(\mathrm{~A})-C_{44}(\mathrm{E})$, on the basis of linear behaviour. All these arguments show that the resulting changes of the averaged values of $C_{11}$ and $C_{44}$, of the averaged values of $C_{11}$ and $C_{44}$, when holes are filled with light metalloid atoms, are rather weak and then $\left\langle C_{11}\right\rangle \mid\left\langle C_{44}\right\rangle$ does not vary at all. This explains the experimental data.

One main effect is the catastrophe for $R=3.6$ atomic units, which is probably balanced by the presence of defects in real materials, and these defects being vacancies which can be occupied by small atoms. This explains the lowering of $C_{11}$ and $C_{44}$ or of Young's modulus and the Poisson ratio as observed [9]. Because of the additivity rule, the adjunction of suitable components modifies these properties in an understandable way [9].

3.3 NORMAL MODES. - The LEC are useful to describe strictly localized modes as in this paper. On the other hand the long wavelength modes are deduced from the average of the LEC's. Moreover the shell structure of the ML model leads us to expect « shell modes " where the different shells keep their individuality. For instance, one can localize a motion in a shell or one can consider the relative motion of shells turning around each other as a block. This last concept is due to Landau [17] when speaking of superfluidity, because of the low frequency of these " rotons " which involve rotation of a large mass of matter. This concept has been actualized by Heritier et al. [18], which coined the name solidons when speaking of normal modes of amorphous solids or of viscous liquids. Here one cannot but focus its attention on the catastrophe region at $R=3.6$ atomic units where the amorphous solid is weaker, and where such a motion will be easier. Other normal modes exist and can be quite useful in order to interpretate glassy melting, with the appearance of more or less large free clusters.

\section{Concluding remarks.}

As a concluding remark we wish to emphasize the very special chaos of the ML structure. This disorder is revealed by the analysis of LEC data. The geometrical distribution of sites shows the alternance of frustrated and unfrustrated shells i.e. local layers in the general meaning of the term. Moreover, it must be said there is another cause of chaos which has to be taken into account. In more realistic macroscopic materials variations from this ML structure are admitted as well as this ML structure because they lead to nearly the same energy per site, they are obtained by a hopping motion of atoms into big holes, i.e. are very close to the ML structure [3]. This packet of states leads to a broadening of the bands, but does not change the main results because no more than $1 \%$ of the sites, at most are shifted. Thus the alteration of LEC cannot be very strong. There is only a further modulation of the 5 typical categories. Finally, the general stability of the ML structure is due to its "self similarity ": far from the centre the structure is quite similar to that at the centre.

\section{Acknowledgments.}

The authors wish to thank Professor T. Egami of Pennsylvania University for fruitful discussions and a useful preprint and Professor J. Friedel for interesting suggestions. 


\section{References}

[1] Cahn, R. W., J. Physique Colloq., to be published.

[2] Gilman, J. J., J. Appl. Phys. 46 (1975) 1625.

[3] Mercier, D. and Levy, J. C. S., Phys. Rev. B 27 (1983) 1292.

[4] Egami, J. and Srolovitz, D., J. Phys.F 12 (1982) 2141.

[5] Maeda, K. and Takeuchi, S., J. Phys. F 8 (1976) 2283.

[6] BoRn, M. and HuANG, K., Dynamical Theory of Crystal Lattices (Clarendon Press, Oxford) 1954.

[7] Levy, J. C. S. and Mercier, D., J. Appl. Phys. 53 (1982) 7709 ;

Lormand, G. and Perez, J., J. Physique Colloq. to be published.

[8] Czjzex, G., Fink, J., Gotz, F., Schmidt, H., Coey, J. M. D. and Rebouillat, J., Phys. Rev. B 23 (1981) 2513.

Fert, A. and Campbell, I. A., J. Phys. F 8 (1978) 257.

[9] Chen, H. S., Rept Progr. Phys. 43 (1980) 354.

[10] Srolovitz, D., Egami, T. and Vitek, V., Phys. Rev. B 24 (1981) 6936.

[11] Levy J. C. S., Surf. Sci. 104 (1981) 1.

[12] Cargill, G. S., III, Solid State Phys. 30 (1975) 227.

[13] Farges, J., Raoult, B. and Torchet, J., J. Chem. Phys. 59 (1973) 3454;
Farges, J., Thesis, Université Paris-Sud (1977).

[14] Muhlbach, J., Sattler, K., Pfau, P. and Recknagel, E., Phys. Lett. 87A (1982) 415-418.

[15] Echt, O., Reyes Flotte, A., Knapp, M., Sattler, K. and Recknagel, E., Ber. Bunsenges. Phys. Chem. 86 (1982) 860.

[16] Valentin, L., Physique Subatomique (Edited by Hermann Press, Paris) 1975.

[17] Landau, L. and Lifshitz, E., Physique Statistique (Ed. MIR, Moscow) 1973.

[18] Heritier, M., Montambaux, G. and Lederer, P., J. Physique 40 (1979) 4493.

[19] Kleman, M., J. Physique 43 (1982) 1389.

[20] Bellessa, G. and Bethoux, O., Phys. Lett. 62A (1977) 125 ; and Bellessa, G., Private communication.

[21] Levy, J. C. S., submitted to J. Physique.

[22] Harris, R., Plischke, M. and Zuckermann, M. J., Phys. Rev. Letters 31 (1973) 160.

[23] KIrtel, C., Introduction to Solid State Physics, 4th edition (John Wiley and Sons) 1971.

[24] Polk, D. E., Acta Metall. 20 (1972) 485. 